\title{
VIDEOJUEGOS EDUCATIVOS: TEORÍAS Y PROPUESTAS PARA EL APRENDIZANE EN GRUPO
}

\author{
EDUCATIONAL VIDEO GAMES: THEORIES AND PROPOSALS FOR GROUP LEARNING \\ Natalia Padilla Zea \\ Ing. Informática, Ph.D. Laboratorio de Investigación en Videojuegos y E-learning. \\ Grupo GEDES. Universidad de Granada, España, \\ npadilla@ugr.es \\ César A. Collazos Ordoñez \\ Profesor Titular, Departamento Sistemas, FIET, universidad del cauca \\ ccollazo@unicauca.edu.co \\ Francisco Luís Gutiérrez Vela \\ Ing. Informática, Ph.D. Laboratorio de Investigación en Videojuegos y E-learning. \\ Grupo GEDES. Universidad de Granada, España, \\ fgutierr@ugr.es \\ Nuria Medina Medina \\ Ing. Informática, Ph.D. Laboratorio de Investigación en Videojuegos y E-learning. \\ Grupo GEDES. Universidad de Granada, España, \\ nmedina@ugr.es
}

Fecha de recepción: 11 de octubre de 2011

Fecha de aprobación: 18 de mayo de 2012

\section{RESUMEN}

En este artículo se presenta el uso de video juegos multijugador que manejan actividades colaborativas como un instrumento educativo acorde con la era digital que vivimos, así como las bases que soportan dicha iniciativa. Este tipo de aplicaciones que se denomina Video GamesSupported Collaborative Learning (VGscL), pretende obtener las ventajas que se derivan de los tres pilares sobre los cuales se apoya la utilización de estas aplicaciones: el uso de videojuegos como elemento atractivo y motivador para un amplio porcentaje de la población en edad escolar, el aprendizaje implícito de conceptos ocultos en los retos y actividades del propio videojuego, y la aplicación de técnicas de aprendizaje colaborativo que permiten a los estudiantes desarrollar sus habilidades sociales mientras aprenden y obtienen un aprendizaje más efectivo.

Palabras clave: aprendizaje colaborativo mediado por computadores, aprendizaje basado en juegos. 


\begin{abstract}
This paper presents the usage of multiplayer video games introducing collaborative activities as an educational tool in line with the digital age we are currently living as well as the basis underpinning this proposal. Such applications, called vascL (Video Games - Supported Collaborative Learning) are intended to obtain the benefits derived from the three milestones on which they are based: usage of video games as an appealing and motivating tool for a big school-age population, the implicit learning of concepts hidden in the game challenges and activities, besides the application of collaborative learning techniques that allow students to develop their social skills as they learn and get a more effective learning.
\end{abstract}

Keywords: computer-supported collaborative learning, game-based learning.

\title{
INTRODUCCIÓN
}

En un siglo cuando la tecnología ocupe gran parte del mercado tanto profesional como personal, sólo era cuestión de tiempo que la tecnología inundara todos los rincones de la vida cotidiana, incluida por supuesto, la enseñanza. Y es que desde hace algunos años, contamos con iniciativas como los centros TIC, Escuela 2.0, y mochilas digitales [3, 4], que impulsan la alfabetización tecnológica de niños. Estas iniciativas proporcionan, entre otras cosas, facilidades para la formación del profesorado, dotación de ordenadores para los centros escolares y los propios estudiantes, fomento para la elaboración de contenidos educativos digitales y, en definitiva, incorporación plena de los recursos multimedia en la educación. Sin embargo, estas iniciativas no están exentas de controversia y el debate se ha iniciado.

Un caso particular de tecnología son los videojuegos. Su uso, desde un punto de vista general, está rodeado de numerosas opiniones encontradas que no dejan que la sociedad se ponga a favor o en contra de ellos. Lo que sí es indudable es que la industria de los videojuegos ha experimentado un crecimiento exponencial en los últimos años, y que el rango de edades a los cuales están dirigidos, se ha incrementado hasta cubrir todo el espectro posible. Por lo tanto, se presenta la disyuntiva Videojuegos SI Vs. Videojuegos NO, sobre todo porque los menores parecen estar muy interesados en estos dispositivos que muchas veces los adultos no pueden manejar.

En consecuencia, si las instituciones competentes impulsan la introducción de tecnología en las aulas y con el supuesto muy antiguo de que una buena forma de aprender es la utilización del juego durante el aprendizaje, ¿se podrá conseguir que los estudiantes mejoren su aprendizaje, usando videojuegos? Esta pregunta, de difícil respuesta, es la que se discutirá en este trabajo.

En el punto 2, se presenta la brecha que existe entre el docente y el estudiante cuando se trata de emplear tecnología en el aprendizaje. Es lo que se ha denominado nativos digitales (estudiantes), 
frente a los inmigrantes digitales (profesores). En el punto 3, se analizan algunas de las ventajas e inconvenientes que en la literatura se atribuyen a los videojuegos. A continuación, en el punto 4 se presentan algunas experiencias representativas desarrolladas con técnicas de aprendizaje basado en juegos (GBL), y en la sección 5, conceptos generales sobre el aprendizaje colaborativo soportado por computador (CSCL). A partir de estas bases, en el punto 6 se presenta el paradigma de aprendizaje Video Games - Supported Collaborative Learning (VGSCL), que trata de paliar los inconvenientes detectados en videojuegos educativos clásicos. Por último, en el punto 7 se presenta una breve discusión a modo de conclusión.

\section{NATIVOS DIGITALES FRENTE A INMIGRANTES DIGITALES}

Prensky M. [20], comenta que los escolares ya no encajan bien en el sistema educativo tal como se concibió, debido a que han crecido en un entorno rodeado de ordenadores, videojuegos, reproductores de música digital, videocámaras, teléfonos móviles y toda clase de juguetes y herramientas de la era digital. Por lo tanto, se les puede considerar Nativos Digitales, que significa que su lengua nativa es el lenguaje digital de los ordenadores, videojuegos e Internet.

Como contrapunto, en ese mismo documento, Prensky M. [20] presenta la figura del profesor como Inmigrante Digital, porque no ha nacido y crecido rodeado de estos dispositivos, pero en algún momento ha tomado contacto con ellos y trata de manejarlos de la mejor forma posible. Se produce entonces, una analogía con el proceso de aprendizaje de un idioma diferente al propio, cuyo conocimiento va a otra parte del cerebro diferente al conocimiento nativo.

A partir de esta realidad, se presentan algunos inconvenientes al aplicar las iniciativas anteriormente comentadas, que se agudizan cuando se intenta avanzar un paso más y utilizar los videojuegos como herramienta educativa.

\section{VENTAUAS E INCONVENIENTES DE LOS JUEGOS EDUCATIVOS}

A partir de los conceptos de nativos e inmigrantes digitales, es lógico pensar que una parte del profesorado pueda sentirse inseguro en cierta forma, a la hora de incorporar tecnologías de juego y aprendizaje en sus clases, sobre todo cuando desde diferentes sectores han resaltado numerosos inconvenientes a los videojuegos, tales como violencia, adicción, aislamientos o sexismo, que se comentan a continuación. Sin embargo, estos temores no están del todo justificados, tal como se muestra a continuación [9, 10, 17].

Existen videojuegos de diversas temáticas, y en numerosas ocasiones, se relacionan directamente con aquellos de contenido violento. Dejando al margen la necesidad de intervención de los padres en este tipo de cuestiones y el hecho de que existe una clasificación por edad de los 
videojuegos, se puede decir que no existe evidencia científica que demuestre que el uso de este tipo de videojuegos desencadene conductas agresivas o patológicas en los jugadores. En el sentido contrario, hay autores que indican que este tipo de juegos permiten descargar la tensión durante el juego, produciendo efectos tranquilizadores que disminuirían la probabilidad de que el jugador cometiese actos violentos. Otro factor por considerar es el estudio acerca de la dirección de esta influencia: Les el videojuego el que desencadena conductas violentas o son jugadores violentos los que acceden a este tipo de contenidos? [12].

Por otra parte, al ser elementos atractivos, aparece la amenaza de adicción en los jugadores. Sin embargo, no se puede confundir adicción con abuso. Es cierto que un jugador que recibe un juego nuevo jugará más al principio hasta que explore los retos y el funcionamiento del juego. Una vez superada esta fase, adopta un comportamiento normal frente al videojuego y no supone un impedimento para realizar otras actividades.

Otra característica negativa que se ha adjudicado a los videojuegos, es la referente al aislamiento, argumentando que los videojuegos hacen que los jugadores se aíslen del mundo y se conviertan en seres asociales. Si bien los videojuegos permiten que el jugador juegue sólo, los juegos preferidos por buena parte de los usuarios, son aquellos con los cuales juegan muchos jugadores, ya sea de forma competitiva o colaborativa. Son numerosos los juegos que favorecen la socialización al presentar competiciones, problemas o retos que hay que resolver en equipo,sobre todo a raíz de la aparición de juegos on-line o los nuevos dispositivos de interacción (por ejemplo, la Wii). Incluso, cuando hay varios jugadores y sólo uno está en los mandos en un momento dado, los demás intentan aportar soluciones, ayudarlo en el proceso, dar sus opiniones, etc. En definitiva, se establecen vínculos entre los jugadores en torno del juego, ya sean dentro del propio juego para superar los retos o como elemento adicional en el ambiente en donde se desarrolla.

Además, es cierto que hay juegos que comercialmente se diseñan de forma específica para niños y otros para niñas. Este sexismo en los videojuegos, que efectivamente se ha dado, ya ha comenzado a resolverse. Aunque con intenciones diferentes, empresas de videojuegos y movimientos feministas, más o menos radicales, reivindican juegos en los cuales los personajes no estén estereotipados y las tramas fomenten valores de colaboración y aventura, con el objeto de captar la atención de un público más amplio y romper las barreras asociadas con el sexismo.

Por último, y más en consonancia con el tipo de videojuegos en estudio, existe la creencia generalizada de que el uso de videojuegos provoca efectos negativos en el rendimiento académico. Sin embargo, parafraseando a Begoña Gross [10] "ya nadie duda que se puede aprender jugando". Es más, no sólo no es un inconveniente, sino que es un elemento motivador que favorece la atención de los niños, su desarrollo cognitivo, habilidades psico-motrices e interés por el aprendizaje, entre otros. Numerosos estudios avalan ya la posibilidad de introducir videojuegos en ambientes educativos con resultados altamente beneficiosos $[6,12]$. 
En realidad, al contrario de perjudicarles, los videojuegos educativos permiten a los niños desarrollar habilidades espaciales y psicomotrices, mejoran la coordinación cerebro-mano, despertar los reflejos y favorecer las capacidades de planificación y desarrollo de estrategias [21], sin olvidar uno de los aspectos más importantes que es incrementar la motivación del estudiante y por consiguiente, ayudar al profesor para mantener su atención.

\section{APRENDIZAJE BASADO EN JUEGOS (GAME - BASED LEARNING)}

El aprendizaje basado en juegos Game - Based Learning (GBL), y en particular, el aprendizaje basado en juegos digitales Digital Game - Based Learning (DGBL), se ha utilizado en diversas experiencias y ha dado buenos resultados. A continuación, se comentan algunas de estas experiencias, clasificadas en función del tipo de juego utilizado (comercial, educativo o serio):

Tabla 1. Videojuegos comerciales conocidos y sus beneficios formativos [1 1]

\begin{tabular}{|l|l|}
\hline \multicolumn{1}{|c|}{ JUEGO } & \multicolumn{1}{c|}{ BENEFICIOS FORMATIVOS } \\
\hline Age of Empires II & Historia, estrategia, administración de recursos \\
\hline Age of Mythology & Mitología, estrategia y administración de recursos \\
\hline Bioscopia & Zoología, biología celular, biología humana, botánica y genética \\
\hline Chemicus & Química \\
\hline Civilization III & Planificación y resolución de problemas \\
\hline $\begin{array}{l}\text { Making history: The calm and } \\
\text { the storm }\end{array}$ & Historia, Segunda Guerra Mundial, gestión económica y negociación \\
\hline $\begin{array}{l}\text { Nancy Drew: Message in a } \\
\text { Haunted Mansion }\end{array}$ & Investigación, deducción y resolución de rompecabezas \\
\hline Oregon Trail & $\begin{array}{l}\text { Historia, geografía, matemáticas, razonamiento lógico, estrategia, administración } \\
\text { de recursos y lectura }\end{array}$ \\
\hline Pharaoh & Civilización egipcia, estrategia y administración \\
\hline Reader Rabbit & Lectura y escritura \\
\hline $\begin{array}{l}\text { Return if the Incredible } \\
\text { Machine Contraptions }\end{array}$ & Habilidades para la resolución de problemas y física \\
\hline Roller Coast Tycoon 3 & Administración, energía cinética y potencial \\
\hline Toontown & Colaboración social \\
\hline $\begin{array}{l}\text { Where in Time is Carmen } \\
\text { Santiago }\end{array}$ & Descubrimiento y lógica \\
\hline World of Warcraft & Aprendizaje colaborativo \\
\hline Zoombinis Logical Journey & Lógica y álgebra \\
\hline
\end{tabular}


- Existen distintas iniciativas que han utilizado Videojuegos Comercialespara entrenar habilidades o fomentar valores. En este grupo, se destaca, por ejemplo: la experiencia realizada con el juego NBA Live 2007, donde se pretendía incorporar valores del ámbito deportivo a la vida diaria de los estudiantes de segundo curso de primaria [1]. Los chicos jugaron en clase con el videojuego y tomaron consciencia de las implicaciones del trabajo en grupo, las técnicas deportivas y las diferencias entre realidad y ficción. Felicia P. [11], presenta un resumen de otros juegos que pueden usarse en clase (Tabla 1).

- Sin embargo, el principal inconveniente que se encuentra en este tipo de videojuegos, es la gran carga de trabajo que supone para el profesor que debe conocer un juego que permita trabajar los conceptos que necesita, aprender a jugar, buscar los puntos importantes y confeccionar una sesión posterior para reflexionar sobre el juego y obtener los beneficios educativos deseados.

- Otras experiencias se han realizado, utilizando Videojuegos Educativos, es decir, aquellos que se diseñaron específicamente para utilizar en el ámbito de la educación. En este grupo, se destaca la propuesta de Rosa Carro et al. [5], que introduce un proceso de adaptación en el sistema, de tal forma que el juego evoluciona, tomando en consideración la actuación de los estudiantes. En concreto, se propone Ecottons2, un conjunto de juegos que abarca distintas habilidades: contar historias, comparar objetos, realizar sumas, etc. Estos objetivos no se proponen directamente a los estudiantes, sino por medio de una historia con objetivos fantásticos que ellos pueden conseguir, resolviendo los problemas que se les plantean. Cada juego se repite un cierto número de veces, dependiendo de las acciones de los usuarios, de tal forma que si el usuario se equivoca, pueda intentarlo de nuevo. Este tipo de juegos permite introducir recursos no tradicionales en las aulas, pero presentan el inconveniente de que en general, la carga educativa es demasiado evidente, por lo cual se pierde parte del elemento motivacional asociado al uso de videojuegos.

- Un caso especial de videojuegos educativos son los Videojuegos Serios. Estos juegos tienen como principal característica la aportación de un valor añadido al proceso lúdico que suele estar relacionado con aspectos como la concienciación, la denuncia social o la política. También es habitual encontrarlos como medio de simulación o entrenamiento en habilidades complejas o peligrosas.

- Son juegos donde hay una clara relación con la realidad y no se entiende el uso de mundos fantásticos, sino que se realiza un vínculo entre el mundo virtual y el real.

- Un ejemplo de este tipo de juegos, es el trabajo realizado por Anacleto et al. [2] referente a la educación sexual, denominado Whatisit? En este juego, los jugadores deben adivinar una palabra secreta a partir de un conjunto de pistas, de tal forma que cuanto antes acierten, mayor será la puntuación. Este tipo de juegos tiene más éxito que los anteriores, debido en opinión personal, al mayor protagonismo de la parte lúdica.

\section{APRENDIZAJE COLABORATIVO SOPORTADO POR COMPUTADOR}

Como elemento adicional, en los videojuegos educativos que se proponen, aparecen actividades de tipo colaborativo, de tal forma que se aproveche la componente social intrínseca en todo ser 
humano, para conseguir ventajas adicionales durante el proceso de aprendizaje. Como se sabe, el aprendizaje colaborativo favorece el aprendizaje individual y fomenta las habilidades sociales. Diversos estudios han comprobado que los estudiantes que trabajan de forma colaborativa, desarrollan mejores actitudes frente al proceso de aprendizaje, dedican más tiempo a la tarea de aprender, son más tolerantes, escuchan más las opiniones de los demás y tienen habilidades de negociación [18]. En realidad, aprenden durante la construcción del conocimiento compartido [18]. Dependiendo de la manera como los estudiantes se comporten, se promueve el éxito entre ellos, se obstruye su proceso de aprendizaje o no se tiene algún efecto sobre el fracaso o el éxito [14]. Lo cierto es que para trabajar cooperativa o colaborativamente, es necesario aprender a hacerlo. No todo es cuestión de poner en un mismo lugar, un conjunto de personas, sentarlas juntas frente a frente e indicarles que cooperen o colaboren en la realización de una actividad [8].

En el caso de hacer esta tarea colaborativa con la ayuda de un soporte tecnológico, es decir, con un computador, se tiene lo que se denomina aprendizaje colaborativo soportado por computador, Computer - Supported Collaborative Learning (CSCL). Esta metodología de enseñanza / aprendizaje reúne las mismas características y cualidades del aprendizaje colaborativo tradicional, pero incluye un elemento motivador asociado a la tecnología. Además, desde el punto de vista del profesor, el uso de computadores como herramienta de aprendizaje, permite realizar un seguimiento del proceso más detallado, porque las distintas herramientas y aplicaciones pueden incorporar el registro de las actividades. De esta forma, el profesor puede revisar el proceso que cada estudiante ha seguido en su aprendizaje, y consultar las puntuaciones y errores cometidos.

Para facilitar este proceso de revisión, existen algunos proyectos, como el que se presenta en Padilla et al [19] que permite al profesor obtener información en tiempo real de los acontecimientos más importantes de cada estudiante. Para ello, el profesor dispone de un dispositivo móvil (PDA), que le permite desplazarse por el aula para atender y supervisar a sus estudiantes. En esta PDA, el profesor ve una representación de todos los puestos del aula con su configuración real y tiene información de qué alumno está sentado en cada puesto. Además, cada vez que ocurre un evento relevante en alguno de los ordenadores de sus estudiantes, la PDA emite una alerta que se representa en clave de colores para que el profesor dé un vistazo y pueda conocer la situación de todos los chicos. De esta forma, si hay uno que no está ejecutando el programa que debería o no es capaz de resolver un problema, el puesto correspondiente se pondrá de color rojo en la PDA del profesor, y permite así, una atención rápida a los estudiantes en dificultades. De forma similar, si un estudiante ha terminado su tarea, el puesto correspondiente se coloreará de azul, y el profesor podrá dar la orden de que comience una nueva aplicación. Esta orden puede darla desde su dispositivo móvil sin necesidad de desplazarse a su mesa ni a la mesa del estudiante. Para facilitar la revisión de conocimientos adquiridos, tanto desde la PDA como desde el ordenador del profesor, se puede consultar un gráfico con las puntuaciones obtenidas por cada estudiante o un gráfico con los resultados de todos los alumnos. 


\section{APRENDIZANE COLABORATIVO SOPORTADO POR VIDEOJUEGOS}

Durante este trabajo, se ha presentado lo que se puede considerar los tres pilares del aprendizaje colaborativo soportado por videojuegos, Video Games - Supported Collaborative Learning (VGSCL), término acuñado para denominar los videojuegos educativos que incluyen actividades colaborativas. Ahora se describirán las principales características que estas aplicaciones deben presentar:

1. Son videojuegos: En la actualidad, existen numerosas aplicaciones que funcionan en un computador o una consola y sirven para enseñar, pero no son videojuegos. Incluso, no son juegos, sino una mera unidad didáctica multimedia que ofrece el mismo procedimiento que un libro de texto tradicional [16]. Esto se traduce en que una vez el niño se da cuenta de este hecho, todas las bondades que a priori se podían obtener, quedan en el olvido. Y es que según la taxonomía de Lepper y Malone [15], un juego educativo debe tener las siguientes características:

a. Desafío: Es necesario crear unos objetivos claros y relevantes para el estudiante. Ofrecer varios niveles de dificultad, información oculta y aleatoriedad. La realimentación debe ser constante, clara y concreta. La actividad que se realiza debe promover sentimientos de competencia en los participantes.

b. Curiosidad: Existe en dos formas diferentes: sensorial y cognitiva. Los efectos audiovisuales, particularmente en los juegos de ordenador, acentúan la curiosidad sensorial. Cuando los aprendices se sorprenden o intrigan por paradojas o información incompleta, se incentiva la curiosidad cognitiva.

c. Control: Se experimenta por medio de sentimientos de autodeterminación y control por parte del aprendiz. Sentimientos de contacto, elección y poder contribuyen al aspecto de control de la experiencia de aprendizaje. Cuando los jugadores afrontan las decisiones, se incrementa su sensación de control personal.

d. Fantasía: Abarca tanto las emociones como los procesos de pensamiento del aprendiz. Las fantasías deben referirse no sólo a las necesidades emocionales, sino que deben proporcionar metáforas y analogías relevantes. Además, las fantasías deben tener una relación directa con el material que se está tratando.

Estas son pues, las características que distinguen una unidad didáctica multimedia de un juego. Cuando se sienta un niño delante del ordenador y se le pregunta qué juego quiere jugar, ¿cuál de las dos modalidades escogería? Seguramente, un tipo de juego concreto. Entonces, parece lógico pensar que cuanto más se parezcan los videojuegos al tipo que el niño elige en la mayoría de las ocasiones, más veces querrá jugar.

2. Son educativos: Esto significa que con este juego, los estudiantes van a aprender cosas. Sin embargo, este aprendizaje se produce de forma implícita, es decir, sin que el niño sea consciente de que está aprendiendo. Por ejemplo: cuando el reto que le plantea al jugador es rescatar a 
sus amigos que ha secuestrado su enemigo. Si, por ejemplo: se le quiere enseñar a distinguir los peces de río y los peces de mar, se le puede proponer que compre a los pescadores un número determinado de peces de río, que cambiará por una pista para continuar el camino. De esta forma, el reto que se le plantea forma parte de la propia dinámica del juego, con lo cual se consigue la motivación necesaria para que el estudiante siga jugando mientras aprende. Al final, se logra que aprenda a distinguir los peces de río de los de mar y además, que adquiera una pista que le va a ser necesaria en la dinámica del juego.

3. Incluyen actividades colaborativas: El número de actividades colaborativas que incluya el juego, depende en gran medida de la edad y desarrollo general de los estudiantes con los cuales se esté trabajando. Así, para niños más pequeños, cuyo aprendizaje debe hacerse de forma más individual, los juegos presentarán menos actividades colaborativas. Por ejemplo: un juego para aprender las vocales tendrá menos actividades colaborativas que un juego para aprender historia. De esta forma, siguiendo con el modelo de Lepper y Malone [15], las características que debe presentar este tipo de juegos son:

a. El desafío debe plantearse al grupo, de tal forma que el objetivo del juego sea común a todos. Se puede indicar en una barra, el grado de dificultad del objetivo común, según como vayan avanzando los jugadores del equipo.

b. El control puede residir en cada usuario particular o estar compartido por el grupo, de tal forma que la influencia de las acciones de unos sobre otros se refleje durante la partida o sólo en los resultados. Dependerá del tipo de juego.

c. Es necesario un componente de conciencia de grupo. El jugador debe conocer en todo momento, quiénes son los miembros de su equipo, si están activos, qué están haciendo (sobre todo, si afecta al grupo), si han terminado el nivel o si necesitan ayuda.

En este sentido, Johnson and Johnson [13] proponen un conjunto de elementos que debe darse para que este tipo de actividades sea lo más beneficioso posible. Sus componentes son:

a. Interdependencia positiva: Los estudiantes son conscientes de que son un equipo, de forma que el éxito o el fracaso del grupo representa su propio éxito o fracaso personal. Los objetivos del grupo deben lograrse por medio del trabajo de todos sus miembros.

b. Exigibilidad personal: Cada miembro del grupo debe ser capaz de aportar su conocimiento al grupo y de aprender lo que sus compañeros le aporten, con el fin de beneficiar a todo el grupo: "No vale descansarse sobre los demás".

c. Interacción positiva cara a cara: Se produce durante el proceso de aprendizaje, cuando los alumnos comparten sus conocimientos, discuten distintos puntos de vista, y ayudan al resto con las dificultades.

d. Habilidades interpersonales y de grupo: Los estudiantes deben organizar el trabajo y tomar decisiones, manifestando sus dotes de liderazgo, conciliación, etc. 
e. Autoanálisis del grupo: El grupo debe autoanalizarse para saber si su trabajo está siendo efectivo, se alcanzan las metas y se está trabajando en el ambiente adecuado. Esto permite que los miembros del grupo fortalezcan sus habilidades de trabajo y fomenta el compromiso de todos con los objetivos comunes.

\section{DISCUSIÓN}

En los últimos años, se ha producido un cambio en las actitudes y aptitudes de los escolares y es necesario que los procesos de enseñanza / aprendizaje se adapten a estas nuevas características. Si bien es cierto que a veces el profesorado se siente en desventaja frente a los conocimientos y habilidades tecnológicas de los estudiantes, no se puede olvidar que el papel del docente sigue siendo clave en su proceso de aprendizaje, por lo cual se está haciendo esfuerzos para dotar al profesor de herramientas que le faciliten su labor sin que se pierda de vista los nuevos requerimientos de los estudiantes. Es cierto que este papel puede haber sufrido una evolución y ya no sea tanto la persona que dicta el conocimiento como el mentor que guía al alumno en su proceso [7], pero en cualquiera de los casos, es indiscutible que el estudiante necesita ser tutelado, ya que hasta los cursos de enseñanzas superiores que se realizan de forma virtual, están apoyados por tutores que ayudan a los estudiantes en el proceso de aprendizaje.

Lo que en este trabajo se ha planteado es un mecanismo que despierte de forma efectiva la motivación del estudiante convertido en jugador, de tal forma que se pueda aprovechar los ratos de ocio del niño para que aprenda algunos conceptos de su currículo. Por supuesto, los videojuegos educativos con actividades colaborativas no pretenden más que servir de apoyo y refuerzo a los contenidos adquiridos en el plantel.

En este proceso de desarrollo de aplicaciones, VGSCL, actualmente está finalizando un videojuego cuyo contenido educativo pretende reforzar los conocimientos impartidos sobre la nutrición en sexto de primaria. En este juego, los estudiantes son asesores nutricionales cuyo objetivo es convertirse en parte del equipo de trabajo de algunos de los personajes preferidos por los adolescentes, tales como cantantes o futbolistas. Una parte fundamental de este videojuego, es la evaluación del proceso colaborativo que ocurre durante el juego, con el objeto de intervenir en el proceso de aprendizaje para poder mejorarlo.

\section{AGRADECIMIENTOS}

Este trabajo ha sido financiado por el Ministerio de Ciencia e Innovación de España como parte del proyecto VIDECO (TIN2011-26928) y el Vicerrectorado de Política Científica e Investigación de la Universidad de Granada. 


\section{REFERENCIAS BIBLIOGRÁFICAS}

[1] Aguayos Rausa J., Almazán L., Bernat A., Campos F., Cárdenas J.J., Vilella X., y Gros Salvat B., (2000). Un espacio para la simbiosis. En: Cuadernos de Pedagogía. Vol. 291, pp. 66-69.

[2] Anacleto J.C., Villena J.M.R., Silva M.A.R., and Fels S., (2010). Culturally sensitive computer support for creative co-authorship of a sex education game. En: Yang H. S., Malaka R., Hoshino J., Han J. H., (eds.) Entertainment Computing-ICEC 2010. LNCS, Vol. 62 (43), pp. 302-307, Springer, Heidelberg.

[3] BOE 20. Centros TIC (2007). En: http://mww.boe.es/boe/dias/2008/01/23/pdfs/A0446704501.pdf (mayo de 2012).

[4] BOE 188. Escuela 2.0 (2009). En: http://mww.boe.es/boe/dias/2009/08/05/pdfs/ BOE-A-2009-13026.pdf (mayo de 2012).

[5] Carro R., Breda A.M., Castillo G., y Bajuelos A.L., (2002). Generación de juegos educativos adaptativos. En: III Congreso Internacional de Interacción Persona-Ordenador. pp. 1664-171.

[6] Ceranoglu T.A., (2010). Video games in psychotherapy. Review of General Psychology. Vol. 14 (2), pp.141-146.

[7] Collazos C.A., Guerrero L., y Vergara A., (2001). Aprendizaje colaborativo: Un cambio en el rol del profesor. Proceedings of the $3^{\text {rd }}$ Workshop on Education on Computing, Arenas, Chile.

[8] Collazos C.A., Ochoa S.F., y Mendoza J., (2007). La evaluación colaborativa como mecanismo de mejora de los procesos de evaluación del aprendizaje en un aula de clase. Revista Ingeniería e Investigación, Vol. 27 (2), pp.72-76.

[9] García Fernández F. (2005). Videojuegos: Un análisis desde el punto de vista educativo. En: http://www.irabia.org/departamentos/nntt/proyectos/futura/futura06/Analisis_educativo. pdf (mayo de 2012).

[10] Gros B., (2000). La dimensión socioeducativa de los videojuegos. Edutec-e: Revista Electrónica De Tecnología Educativa, Vol. 12.

[11] Felicia P., (2009). Videojuegos en el aula. Manual para docentes. Bélgica, European school net, $46 \mathrm{p}$.

[12] Ferguson C.J., (2010). ¿Blazing angels or resident evil? ¿Can violent video games be a force for good? Review of General Psychology, Vol. 14 (2), pp. 68-81. 
[13] Jonhson D.W., y Jonhson R.T., (1994). Learning together. En: S. Sharan (Ed.), Handbook of cooperative learning methods. Connecticut: Greenwood Press.

[14] Jonhson D.W., y Jonhson R.T., (1998). Cooperative learning and social interdependence theory. En: Tindale R., Heath L., Edwards J., Posavac E., Bryant F., Suzrez-Balcazar Y., Henderson-King E. \& Myers J. (Eds.). Theory and research on small groups. pp. 9-36. New York: Plenum.

[15] Lepper M.R., and Malone T.W. (1987). Intrinsic motivation and instructional effectiveness in computer-based education. En: R. E. Snow, \& M. J. Farr (Eds.), Aptitude, learning, and instruction: III. Cognitive and affective process analysis. pp. 255-286. Hillsdale, New Jersey: Erlbaum.

[16] McFarlane A., Sparrowhawk A., and Heald Y., (2002). Report on the educational use of games: An exploration by TEEM of the contribution which games can make to the education process. Consultado en mayo de 2012. En: http://www.mendeley.com/research/report-onthe-educational-use-of-games-an-exploration-by-teem-of-the-contribution-which-gamescan-make-to-the-education-process/

[17] Méndiz A., Pindado J., Ruíz J., y Pulido J. M., (2002). Videojuegos y educación: Una revisión crítica de la investigación y la reflexión sobre la materia. Ministerio de Educación y Ciencia de España. En: http://ares.cnice.mec.es/informes/02/documentos/indice.htm (mayo de 2012).

[18] Mendoza Barros P., and Galvis Panqueva A., (1998). Juegos Multiplayer: Juegos colaborativos para la educatión. En: Informática Educativa, Santafé de Bogotá: UNIANDES - LIDIE, Vol. 11 (2), pp. 223-239.

[19] Padilla N., Rodríguez F.J., Cabrera M., and Morales J., (2010). Using the ACOLEP-MD architecture: Application to Edutainment. Procedia Social and Behavioural Sciences Journal, Vol. 2 (2), pp. 2197-2201.

[20] Prensky M., (2001). Digital natives, digital immigrants. En: On the Horizon Vol. 9 (5), NCB University Press.

[21] Valiño G., (2002). La relación juego y escuela: Aportes teóricos para su comprensión y promoción. Revista Conceptos, Vol. 77 (2).

[22] Weeb N.M., (1996). Group processes in the classroom. D.C. hand book of educational psychology. New York: McMillan. 